\title{
Angehörigenarbeit bei Personen mit substanzbezogenen Störungen
}

\author{
Der Community Reinforcement and Family Training (CRAFT)-Ansatz
}

\section{G. Bischof ${ }^{1}$ \\ J. Freyer $^{2}$}

\author{
Working with Relatives of Individuals with Substance Related Disorders: \\ The Community Reinforcement and Family Training (CRAFT) Approach
}

\section{Zusammenfassung}

In Deutschland gelten nach Schätzung der Deutschen Hauptstelle für Suchtfragen 5 - 7 Millionen Angehörige von Alkoholabhängigen als von der Abhängigkeit unmittelbar mit betroffen. Traditionelle Ansätze in der Suchtkrankenhilfe fokussierten in der Vergangenheit unter dem Modell der „Co-Abhängigkeit“ auf konfrontativen Strategien, deren Wirksamkeit empirisch nicht hinreichend belegt werden konnte. Unter der Bezeichnung „Community Reinforcement and Family Training“ CRAFT wurde in den USA eine nichtkonfrontative Strategie für Angehörige von Suchtkranken entwickelt, die nach einer Metaanalyse zu den wirksamsten Interventionsansätzen im Bereich der Suchtkrankenhilfe zählt. Dabei werden motivationale Strategien und behaviorale Interventionen kombiniert eingesetzt. Dieser Artikel gibt einen Überblick zu Grundannahmen, den verschiedenen Modulen und Wirksamkeitsnachweisen des CRAFT-Ansatzes.

\section{Schlliisselwörter}

Angehörige $\cdot$ Alkohol $\cdot$ Intervention $\cdot$ Sucht

\section{Abstract}

In Germany, an estimated 5-7 million relatives of alcohol-dependent individuals are assumed to be directly affected. In the past, traditional concepts within the treatment system, based on the concept of "co-dependency", have focused on confrontational approaches which have not proven to be effective. Under the heading "Community Reinforcement and Family Training" (CRAFT), a non-confrontational strategy for significant others of subjects with alcohol problems and chemical dependency has been developed in the US. According to a meta-analysis, CRAFT is among the most effective approaches in the field of addiction treatment. It combines motivational and behavioral interventions. The present paper provides an overview on basic principles, the different elements and study results of CRAFT.

Key words

Relatives $\cdot$ alcohol $\cdot$ intervention $\cdot$ addiction

\section{Einleitung}

In Deutschland gelten nach Schätzung der Deutschen Hauptstelle gegen Suchtgefahren 5-7 Millionen Angehörige von Alkoholabhängigen als von der Abhängigkeit unmittelbar mit betroffen. Bei Angehörigen von Menschen mit substanzbezogenen Störungen zeigen sich erhöhte Raten stressbedingter Erkrankungen und psychosozialer Beeinträchtigungen [1-4].
Bislang liegen nur wenige Studien zur Nutzung familiärer Prozesse in der Suchtkrankenversorgung vor. Meist handelt es sich dabei um Stichproben, bei denen die Familie nach Behandlungsbeginn in die Therapie mit einbezogen wurde. Eine Reihe qualitativer Befunde aus der Perspektive der systemischen Therapie beschäftigte sich unter dem Aspekt der „Co-Abhängigkeit“ insbesondere mit pathogenen Faktoren im Sinne von aufrechterhaltenden Funktionen des familiären Systems für das Abhängig-

Institutsangaben

${ }^{1}$ Klinik für Psychiatrie und Psychotherapie, Arbeitsgruppe S:TEP (Substanzmissbrauch:

Epidemiologie, Prävention), Universität zu Lübeck

${ }^{2}$ Institut für Epidemiologie und Sozialmedizin, Ernst-Moritz-Arndt-Universität Greifswald

Korrespondenzadresse

Dr. Gallus Bischof · Universität zu Lübeck, Klinik für Psychiatrie und Psychotherapie, Arbeitsgruppe S:TEP .

Ratzeburger Allee 160 ·23538 Lübeck·E-mail: gallus.bischof@ukl.uni-luebeck.de

Bibliografie

Suchttherapie 2006; 7: 52 - 57 c Georg Thieme Verlag KG Stuttgart · New York

DOI 10.1055/s-2006-926754

ISSN 1439-9903 
keitsgeschehen [5]. Eine empirische Prüfung der aus diesen Konzepten abgeleiteten Schlussfolgerungen steht bislang jedoch aus. Weitere ältere Studien legten den Fokus auf die Verbesserung psychosozialer Parameter der Angehörigen, ohne Entwicklungsprozesse des/der Abhängigen in Betracht zu ziehen [6].

Verhältnismäßig konsistente Befunde liegen aus Studien zur zusätzlichen Einbeziehung von Familienangehörigen in die professionelle Behandlung von Suchtmittelabhängigen vor, wobei aufgrund der eher globalen Analysen kaum Aussagen über die Wirkung spezifischer familiärer Prozesse abgeleitet werden können [7]. Verhältnismäßig gut belegt ist der Befund, dass nach Aufnahme einer Behandlung die Einbindung Angehöriger in den therapeutischen Ablauf zu einer Erhöhung regulärer Therapiebeendigungen beiträgt $[8,9]$. Die Bedeutung sozialer Unterstützung für die Aufrechterhaltung der Abstinenz wurde sowohl bei behandelten als auch bei unbehandelten remittierten Alkoholabhängigen in verschiedenen Studien nachgewiesen [10]. Einschränkend ist hier festzuhalten, dass Befunde an behandelten Alkoholabhängigen darauf hinweisen, dass die Nutzbarkeit familiärer Beziehungen geschlechtsspezifisch variiert; so sind alkoholabhängige Frauen zu Behandlungsbeginn häufiger geschieden oder mit einem alkoholabhängigen Partner verheiratet [11] Ähnlich deutliche Geschlechterdifferenzen bezüglich familiärer Variablen konnten auch bei Alkoholabhängigen, welche ohne formelle Hilfen remittierten, festgestellt werden [12].

Die Effizienz von Familientherapie bei der Behandlung von Abhängigkeitserkrankungen konnte für einige Verfahren nachgewiesen werden [13]. Als problematisch ist hierbei anzusehen, dass in einer Reihe von Studien keine randomisierte Zuordnung vorgenommen wurde, weshalb die Ergebnisse aufgrund von Selbstselektionsaspekten einer starken Verzerrung unterworfen sein können. Schwerpunkt verhaltensorientierter Interventionen stellt das Training der Angehörigen zur Verstärkung substanzunabhängiger Aktivitäten dar, wie beispielsweise mittels der verhaltensorientierten Paartherapie [14].

\section{Angehörige und Frühintervention}

Eine Schwierigkeit bei der Analyse des Einflusses von Angehörigen auf den Verlauf von Alkoholabhängigkeit ist in der Beschränkung auf Angehörige, von sich in Behandlung befindenden Personen mit substanzbezogenen Störungen, zu sehen. Insgesamt nimmt jedoch nur eine Minderheit von Alkoholabhängigen Behandlung in Anspruch. Nach Angaben eines großen US-amerikanischen Bevölkerungssurveys hatten in den 12 Monaten vor der Erhebung lediglich 9,9\% der Menschen mit einer Alkoholabhängigkeit oder einem -missbrauch Kontakt zum Alkoholhilfesystem aufgewiesen [15]. Daten einer deutschen Bevölkerungsstudie konnten zeigen, dass lediglich etwa $14,5 \%$ der Menschen mit der lifetime Diagnose einer Alkoholabhängigkeit jemals alkoholbezogene Hilfen in Anspruch genommen haben, während 70,9\% keinerlei Kontakte zum suchtspezifischen Hilfesystem aufwiesen [16]. Auch stellen Angehörige unter den Inanspruchnehmern des Suchthilfesystems weniger als 10\% dar [17]. Gleichzeitig konnten verschiedene Studien nachweisen, dass Familienangehörige bei unbehandelten Alkoholabhängigen einen bedeutsamen Einfluss sowohl auf Ausstiegsprozesse ohne Be- handlung als auch auf die Inanspruchnahme von Behandlung ausübten [18]. Insgesamt jedoch steht den Studien zur Einbindung von Familienangehörigen bei Klienten der herkömmlichen Suchthilfeeinrichtungen nur ein geringer Anteil an methodisch meist problematischen Studien zur Nutzung von Familienangehörigen bei „unmotivierten“ Alkoholabhängigen gegenüber [19].

\section{Der Community Reinforcement Approach and Family Training (CRAFT)}

Umgebungsfaktoren von Menschen mit substanzbezogenen Problemen sind insbesondere in lerntheoretischen Ansätzen bereits seit längerem im Fokus gezielter Interventionen. Einen solchen Ansatz stellt der Anfang der 70er-Jahre entwickelte Community Reinforcement Approach CRA (Gemeindeverstärkungsansatz) dar, der auf dem Konzept basiert, durch gezielte Beeinflussung von Kontingenzen den Verstärkungswert von nichtkonsumierendem Verhalten gegenüber konsumierendem Verhalten zu erhöhen. In neueren Metaanalysen zur Wirksamkeit von Behandlungsansätzen bei alkoholbezogenen Störungen wurde CRA zu den wirksamsten und ökonomischsten Behandlungsansätzen gezählt [20, 21].

Aus dem ursprünglichen Gemeindeverstärkungsansatz, der sich auf den Einfluss unterschiedlicher sozialer Quellen auf den Verlauf substanzbezogener Störungen fokussierte, wurde in den 80er-Jahren das auf Angehörige zugeschnittene „Community Reinforcement And Family Training“ (CRAFT) [22] entwickelt. Die Anwendbarkeit des Ansatzes wurde bislang nicht nur für problematischen Alkoholkonsum, sondern auch für den Bereich illegale Drogen und pathologisches Spielverhalten überprüft.

In diesem Ansatz wird davon ausgegangen, dass Familienangehörige einen wesentlichen Beitrag für die weitere Entwicklung des Substanzkonsums des Betroffenen (Index-Patient, IP) und die Inanspruchnahme von Hilfen leisten können. Diese Annahme wird durch verschiedene Studien zu Prädiktoren von Inanspruchnahme von Hilfen und von unbehandelten Ausstiegsprozessen aus der Alkoholabhängigkeit gestützt [10, 23]. Gegenstand des Trainings ist primär die Vermittlung der zu diesem Zweck als sinnvoll erachteten Fertigkeiten, ergänzt durch weitere Fertigkeiten mit dem Ziel, die Lebensqualität der Angehörigen zu erhöhen. Das Programm ist wiederholt überarbeitet worden und liegt mittlerweile sowohl in Form eines Manuals für Therapeuten [24] als auch in Form eines Selbsthilfebuches vor [25]. Der Ansatz zielt auf Personen, die in unterschiedlicher Beziehung zu dem Substanzmissbraucher stehen wie z.B. Eltern, Partner, Kinder und enge Freunde.

Gestützt aufgrundlagen der Lerntheorie, nutzt CRAFT Verstärkungsstrategien im Gegensatz zu konfrontierenden Techniken. Diese Strategien lassen sich auf unterschiedliche Abstufungen der Veränderungs- und Behandlungsmotivation eines IP zuschneiden [26]. Das Programm orientiert sich zusätzlich an Verfahren wie der motivierenden Gesprächsführung [27]. Dabei wird ein empathischer und nichtbewertender Gesprächsstil fokussiert, der letzte Entscheidungen über die anzustrebenden Veränderungsschritte dem Klienten überlässt. Zu Behandlungsbeginn erfolgt eine detaillierte Erhebung des aktuellen psychosozialen Funktionsniveaus des Angehörigen sowie der Beziehung 
zwischen dem Angehörigen und dem IP mittels standardisierter Erhebungsinstrumente [24].

In 12 Einzelsitzungen werden folgende Module behandelt: 1. Motivieren des Angehörigen, 2. Funktionale Verhaltensanalyse, 3. Strategien gegen Gewalt, 4. Kommunikationstraining, 5. Positive Verstärkung, 6. Nutzung negativer Konsequenzen, 7. Strategien zur Verbesserung der Lebensqualität, und 8. den IP zur Inanspruchnahme von Hilfe motivieren.

\section{Motivieren des Angehörigen}

Bevor mit dem eigentlichen Training begonnen werden kann, sollte durch Vermittlung von Studienergebnissen in allgemein verständlicher Sprache die Zuversicht der Angehörigen erhöht und ein Ausblick auf die zu erwartenden Ergebnisse gegeben werden. Durch Verwendung eines nichtbewertenden, nichtkonfrontativen, empathischen Stils, und die verbale Verstärkung des Angehörigen soll eine lösungs- und problemorientierte Atmosphäre geschaffen werden.

\section{Funktionale Verhaltensanalyse}

Die funktionale Analyse des Substanzkonsums stellt die Grundlage der Intervention dar. Ziel ist, Ansatzpunkte zur Beeinflussung des Konsumverhaltens des IP zu gewinnen, detaillierte Informationen über den aktuellen Stand zu eruieren und eine bessere Vorhersagbarkeit des Konsumverhaltens zu erreichen. Schematisch orientiert sich das Vorgehen an das in der Verhaltenstherapie geläufige SORKC-Schema [28]. Hierfür wird gewöhnlich eine möglichst typische Konsumsituation gewählt. Besonderes Augenmerk wird auf externe und innere Auslöser gelegt; das Konsumverhalten wird ebenfalls detailliert beschrieben. Das Schema enthält weiterhin eine ausführliche Differenzierung in kurzfristig positive Konsequenzen und langfristig negative Konsequenzen des Konsums. In der Erhebung wird dabei dem Angehörigen viel Raum gegeben, Fragen werden offen gestellt. Da die Erhebung der funktionalen Analyse zu einem frühen Zeitpunkt der Behandlung einsetzt, werden Leerstellen in dem Schema zugelassen. Verhaltensänderungen durch den Angehörigen werden in dieser ersten Stufe noch nicht angestrebt und erfordern zunächst das Durchlaufen des zweiten Moduls.

\section{Strategien gegen Gewalt}

Gewalttätige Übergriffe sind ein verbreitetes Problem in Familien und Beziehungen mit einem substanzabhängigen Menschen. Studien, die mögliche konfundierende Variablen kontrollierten, legen eine direkte Assoziation zwischen dem Substanzmissbrauch und gewalttätigen Übergriffen nahe [29, 30]. Es ist belegt, dass viele Opfer gewalttätiger Übergriffe auch nach mehrmaligen Aufenthalten in beschützten Einrichtungen (wie z. B. Frauenhäusern) erneut zu den gewalttätigen Partnern zurückkehren [31]. Entsprechend betont das CRAFT-Programm die Notwendigkeit, möglichst sichere Rahmenbedingungen zu schaffen, wofür ein eigenes Modul bereitsteht.

Ziel des Moduls ist es, zunächst detailliert bisherige Gewalterfahrungen zu erfragen, um Frühwarnsignale und auslösende Bedingungen besser zu erfassen. Dies geschieht erneut über eine funktionale Verhaltensanalyse, welche wiederum die Vorhersagbarkeit aggressiven Verhaltens (z. B. durch Identifikation von Frühwarnsignalen) verbessert. Intendiert wird die Beendi- gung der Gewalt, wobei alternativ ein Notfallplan erstellt wird (z.B. Zufluchtsmöglichkeiten für den Fall eskalierender Gewalttätigkeiten oder legaler Sanktionierungsmöglichkeiten) und im Krisenfall aktivierbare soziale Unterstützungssysteme eingebunden werden.

\section{Kommunikationstraining}

Kommunikationsprobleme sind in Beziehungen mit einem substanzmissbrauchenden Partner weit verbreitet [32]. Grundlage des Moduls zur Verbesserung kommunikativer Fertigkeiten stellt die Erfahrung dar, dass die Verbesserung kommunikativer Fertigkeiten über die gezielte Auseinandersetzung mit dem substanzmissbrauchenden Angehörigen hinaus eine Verbesserung der Lebensqualität in verschiedensten Lebensbereichen ermöglicht. Die Elemente des Kommunikationstrainings werden durch Psychoedukation und in Form von Rollenspielen vermittelt und erinnern an das zum Standardrepertoire der Verhaltenstherapie gehörende Gruppentraining sozialer Kompetenz [33]. Kommunikativen Fertigkeiten wird zugeschrieben, dass sie der Erreichung eigener Ziele dienlich sind und auch einen zentralen Faktor bei der Beeinflussung des Verhaltens des Angehörigen darstellen.

\section{Positive Verstärkung}

In einem nächsten Schritt wird eine Liste von positiven Verstärkern für den missbrauchenden Angehörigen erstellt, welche auch von der an der Intervention beteiligten Person einsetzbar sind (z. B. Massagen, gemeinsame Aktivitäten wie Kino, Essen gehen). Das Ausmaß an Attraktivität für den IP wird eingeschätzt, die Auswahl der im folgenden einzusetzenden Verstärker erfolgt nach den Gesichtspunkten, inwiefern diese für den IP angenehm sind, finanziell überschaubar bleiben, zur sofortigen Anwendung bereitstehen und von der Seite des Angehörigen auch problemlos anwendbar sind. Diese Verstärker werden bei abstinentem/ nichtkonsumierendem Verhalten eingesetzt. Zugleich werden Anzeichen von Substanzkonsum identifiziert und dem Angehörigen der Zusammenhang zwischen abstinentem Verhalten und positiver Verstärkung mitgeteilt. Hierbei ist von besonderer Bedeutung, eine klare Unterscheidung zwischen unerwünschtem Verstärken des konsumierenden Verhaltens (wie in Konzepten zu Co-Abhängigkeit beschrieben) und der Verstärkung von nichtkonsumierendem Verhalten einzuführen. Schließlich erfolgt eine Auswahl des zu verstärkenden Verhaltens des IP; eine solche zu verstärkende Verhaltensweise sollte dem IP Freude bereiten, mit Substanzkonsum unvereinbar sein, häufig auftreten bzw. zukünftig auftreten können und auch für den Angehörigen angenehm sein (z. B. sportliche Aktivitäten, einen Ausflug unternehmen). Der Zusammenhang zwischen Verhalten und Verstärkung wird auch hier dem IP offen mitgeteilt. Um unerwünschte Lernerfahrungen seitens des IP zu vermeiden, indem z. B. der IP für eine gemeinsame Aktivität in angetrunkenem Zustand verstärkt wird, werden in diesem Modul zusätzlich Anzeichen von Substanzkonsum des IP gesammelt.

\section{Nutzung negativer Konsequenzen}

Das Prinzip der Nutzung negativer Konsequenzen bezieht sich sowohl auf die eigene Interaktion mit der substanzmissbrauchenden Person als auch darauf, natürliche Konsequenzen des Substanzmissbrauchs nicht abzumildern (indem z.B. ein alkoholassoziierter Fehltag bei der Arbeit durch den Angehörigen gedeckt wird). In der Interaktion bedeutet die Nutzung negativer Konsequenzen, 
eine „Auszeit“ bei Konsumverhalten einzuführen, d.h. positive Konsequenzen zu entziehen. Diese werden ausgewählt nach der relativen Bedeutung für den IP und der Fähigkeit des Angehörigen, diese ohne größere Schwierigkeiten und ohne Eigengefährdung zeitnah zu entziehen. Die entsprechenden Zusammenhänge werden auch hier der substanzmissbrauchenden Person mitgeteilt. Das Zulassen natürlicher Konsequenzen des Substanzmissbrauchs bedeutet, dass der Angehörige lernt, alles zu unterlassen, was die Konsequenzen des Missbrauchs in irgendeiner Form abmildert. Dieser auch bei Co-Abhängigkeitskonzepten hervorgehobene Ansatz wird in CRAFT explizit lerntheoretisch vermittelt, d.h. es wird nicht postuliert, dass der Angehörige einen Gewinn aus dem Substanzmissbrauch des IP zieht, dass aber eine Verhaltensänderung durch Blockieren aversiver Konsequenzen erschwert wird. Da aufgrund des Zulassens negativer Konsequenzen neue Schwierigkeiten auftreten können, wird in diesem Modul ergänzend das Problemlöseschema sensu D’Zurilla und Goldfried [34] vermittelt und geübt.

\section{Strategien zur Verbesserung der Lebensqualität}

Zusätzlich zu den auf die substanzmissbrauchende Person abzielenden Bestandteilen des CRAFT-Ansatzes, befasst sich ein Modell der Behandlung mit der Verbesserung der Lebensqualität des Angehörigen. Hierbei werden die anfänglich eingesetzten Erhebungsinstrumente herangezogen, um verbesserungswürdige Lebensbereiche des Angehörigen zu identifizieren. Anschließend werden in diesen Bereichen eigene verhaltensbezogene Lebensziele und Strategien erarbeitet. Als Themenbereiche bieten sich diesbezüglich das eigene Konsumverhalten, eigene berufliche Ziele, Umgang mit finanziellen und juristischen Schwierigkeiten und das eigene emotionale Erleben an. Das Modul fokussiert insbesondere auf den psychosozialen Beeinträchtigungen der Angehörigen, mit Schwerpunkt auf Konkretisierung von Zielen und Kriterien für den Grad der erreichten Zielannäherung. Intendiert wird, das soziale Unterstützungssystem der Angehörigen auszuweiten sowie eigenständige und angenehme Aktivitäten ohne den IP zu fördern. Für letzteren Ansatz wird analog zur verhaltenstherapeutischen Behandlung depressiver Störungen [35] eine Liste positiver Aktivitäten erstellt. Ergänzend findet auch hier das bereits erwähnte Problemlöseschema Anwendung.

\section{Den IP zur Inanspruchnahme von Hilfe motivieren}

Zentrales Ziel des CRAFT-Ansatzes ist es, den Angehörigen zur Inanspruchnahme von Behandlung zu motivieren. Hierfür werden parallel zu den CRAFT-Sitzungen Behandlungsmöglichkeiten arrangiert. Motivationale Ansatzpunkte (z.B. Krisen), so genannte „Windows of Opportunity“, werden gezielt angesprochen, wobei als Modellvorstellung das Konzept der „Stages of Change“ [36, 37] vermittelt wird. Zur Motivationssteigerung werden dem IP verschiedene Optionen angeboten, darunter a) Behandlung durch den CRAFT-Therapeuten des Angehörigen oder einer anderen Person, b) Entscheidung über die Intensität der Behandlung und den eigenen Einfluss bezüglich der avisierten Ziele, inklusive der Möglichkeit, nichtsubstanzbezogene Ziele als Bestandteil der Behandlung zu bearbeiten, und c) Vermittlung des Zusammenhanges zwischen nichtkonsumierendem Verhalten und weiteren positiv assoziierten Lebenszielen. Zur Vermittlung der Bestandteile des Moduls an den IP werden die im Kommunikationstraining erworbenen kommunikativen Fertigkeiten eingesetzt. Vor der Thematisierung von Behandlung sollten bereits entsprechende Kontakte zum Suchthilfesystem hergestellt worden sein, um einen schnellen Behandlungsbeginn zu ermöglichen. Weiterhin werden die Angehörigen auch auf mögliche Weigerungen des IP, sich in Behandlung zu begeben und auf einen möglichen Therapieabbruch vorbereitet. Dabei ist von besonderer Bedeutung, dass eine solche Schwierigkeit nicht als globales Scheitern der eigenen Bemühungen aufgefasst wird, sondern in einem supportiven Rahmen das weitere zielorientierte Vorgehen thematisiert wird. Schließlich wird die Notwendigkeit anhaltender Unterstützung auch während der Behandlung und über diese hinaus dargelegt.

Empirische Studien zur Einbeziehung von Angehörigen in die Fruihintervention

Wie oben ausgeführt, zählen nach neueren Metaanalysen die dem CRAFT-Ansatz zugrunde liegenden Prinzipien der Gemeindeverstärkungsansätze zu den ökonomischsten und wirksamsten Interventionen bei substanzbezogenen Störungen [20, 21]. Die verfügbaren kontrollierten Studien zu CRAFT differieren in Abhängigkeit von der fokussierten Substanz (Alkohol, Drogen) und der Altersgruppe (Erwachsene, Jugendliche). Bei diesen Studien wurde üblicherweise als Einschlusskriterien verwendet, dass es sich um nahe Angehörige (i.d. R. Partner oder nahe Verwandte) handeln musste, die regelmäßigen Kontakt zu dem IP (z.B. mindestens 40\% der Freizeit) aufwiesen. Weiterhin mussten die Angehörigen ein Konsummuster beschreiben, welches die Kriterien für das Vorliegen einer Alkoholabhängigkeit sensu DSM-IV [38] erfüllte. Als Ausschlusskriterium wurden gegenwärtige Behandlungsmotivation des IP oder Inanspruchnahme von Behandlung in den letzten drei Monaten, juristische Behandlungsauflagen, eine Vorgeschichte schwerer gewalttätiger Übergriffe in der Beziehung zum IP sowie das Vorliegen einer psychiatrischen Erkrankung seitens des Angehörigen festgelegt. Die Therapeuten in den durchgeführten Studien wiesen einen Universitätsabschluss mit psychologischem Hintergrund auf, waren jedoch bezüglich ihrer therapeutischen Vorerfahrung sehr heterogen.

Eine groß angelegte, vom National Institute on Alcohol Abuse and Alcoholism (NIAAA) geförderte Studie mit insgesamt 130 Teilnehmern verglich die Wirksamkeit von drei verschiedenen Interventionen für Angehörige. Es handelt sich dabei neben CRAFT um die Angehörigengruppe der Anonymen Alkoholiker Al-Anon und die in den USA verbreitete Johnson Institute Intervention.

Die auch in Deutschland bekannten Angehörigengruppen der Anonymen Alkoholiker, Al-Anon, gehen davon aus, dass Angehörige gegenüber dem problematischen Substanzkonsum machtlos sind und sich stattdessen an die 12 Schritte und 12 Traditionen Al-Anons anlehnen sollten. Primär soll eine Stärkung der eigenen seelischen Gesundheit angestrebt werden. Dabei wird davon ausgegangen, dass dies indirekten Einfluss auf den Alkoholkonsum des Partners nehmen kann, indem z.B. das Trinken stützende oder ermöglichende Verhaltensweisen der Angehörigen abgebaut werden. Eine intendierte direkte Beeinflussung der substanzabhängigen Person wird von diesem Ansatz zurückgewiesen bzw. als nicht gangbar betrachtet. Eine ältere Studie [6], welche die Wirksamkeit von Al-Anon untersuchte, entdeckte, dass, als 23 Frauen von behandlungsresistenten Männern nach dem Zufallsprinzip entweder 
der unmittelbaren oder verzögerten Al-Anon Gruppentherapie zugeteilt wurden, die Frauen in der ersten Bedingung gegenüber denjenigen in der zweiten Bedingung größere Verbesserung sowohl bezüglich ihrer Stimmung als auch des Selbstkonzeptes nach 8 Wochen aufwiesen. Jedoch hatten weder in dieser noch in anderen Studien zu Al-Anon die substanzmissbrauchenden Partner Behandlung begonnen oder ihren Substanzkonsum verändert [22].

Ein in Deutschland weitgehend unbekannter Ansatz stellt eine familienbezogene Intervention, welche am Johnson Institute zur Förderung der Inanspruchnahme von Hilfen entwickelt wurde, dar [39]. In dieser Intervention werden Angehörige darauf vorbereitet, die substanzmissbrauchende Person mit ihren eigenen Beobachtungen und Erfahrungen bezüglich des Trinkens und der damit assoziierten Probleme zu konfrontieren. In dieser Konfrontation soll die substanzmissbrauchende Person auf eine unterstützende Art und Weise ermutigt werden, Behandlung in Anspruch zu nehmen. Für den Fall der Nichtinanspruchnahme sollen Sanktionen ausgeübt werden. Während diesem Ansatz historisch der Verdienst zukommt, erstmalig Angehörigen von Personen mit substanzbezogenen Störungen eine aktive Rolle auf den Krankheitsverlauf zugestanden zu haben, muss der paternalistische Ansatz der Intervention insgesamt als ethisch bedenklich betrachtet werden, was auch die Verbreitung des Konzeptes außerhalb der USA eingeschränkt haben dürfte. Trotz der weiten Verbreitung in den USA liegen zudem bislang erst wenige Studien zur Effektivität des Ansatzes vor [19].

Empirische Studien zur Wirksamkeit des konfrontativen Ansatzes nach Johnson haben in nichtkontrollierten Studien ergeben, dass IPs von Teilnehmern eines Johnson Gruppentrainings gegenüber anderen Interventionsbedingungen häufiger Behandlung in Anspruch nahmen, wobei keine randomisierte Gruppenzuordnung vorgenommen wurde [19]. Zugleich ergaben jedoch die meisten Studien, dass viele Familien den konfrontativen Ansatz gegenüber dem abhängigen Angehörigen nicht umzusetzen in der Lage waren. Eine unkontrollierte Studie des Johnson Ansatzes ergab, dass einerseits nur ein geringer Anteil (29\%) der Familienangehörigen die Konfrontation ausführte, dass aber die konfrontierten IPs deutlich häufiger eine Therapie in Anspruch nahmen und auch länger in dieser verweilten [40]. Problematisch an diesem Ergebnis ist, dass sich die gefundenen Resultate aufgrund von Selbstselektionsprozessen nicht unmittelbar auf die Wirkung des Konfrontationsansatzes zurückführen lassen. Allerdings stimmen die Ergebnisse mit Bevölkerungsstudien überein, nach denen sozialer Druck eine bedeutsame Größe für die Inanspruchnahme therapeutischer Hilfen darstellt $[41,42]$.

In einer Studie, welche die Effizienz der drei dargestellten familienbezogenen Interventionsansätze überprüfte, wurden 130 Angehörige von Alkoholabhängigen randomisiert den drei unterschiedlichen Bedingungen zugeordnet [19]. Unter allen Bedingungen erhielten die Angehörigen jeweils 12 Stunden Beratung bzw. Training. Eine Outcome-Erhebung nach 12 Monaten überprüfte die Raten der Inanspruchnahme formeller Hilfen, die Qualität der Beziehung sowie das psychosoziale Funktionsniveau der Angehörigen. Bei der Nacherhebung, mit einer Ausschöpfungsquote von $94 \%$, ergaben sich deutliche Unterschiede zwischen den Interventionsansätzen. In der CRAFT Gruppe konnten die IPs in 64\% der Fälle in eine Behandlung überwiesen werden, während die Inan- spruchnahme von Hilfen in der Johnson Bedingung mit 30\% und der Al-Anon Bedingung mit 13\% deutlich geringer ausfielen. Ana$\log$ zu früheren Studien wurde die Konfrontation der substanzmissbrauchenden IPs von 70\% der Angehörigen der Johnson Gruppe nicht durchgeführt, und 75\% der konfrontierten IPs konnten durch die Konfrontation nicht zur Aufnahme einer Behandlung motiviert werden. Daraus kann gefolgert werden, dass Konfrontation keinen hinreichenden Grund für die Inanspruchnahme von Hilfsangeboten darstellt. In allen drei Interventionsbedingungen ergaben sich ähnliche Verbesserungen hinsichtlich der Beziehungsqualität und dem psychosozialen Funktionsniveau der Angehörigen.

Eine vergleichbare Studie bei Drogenmissbrauchern und -abhängigen mit dem Schwerpunkt Cannabis-/Kokainkonsum ergab erneut eine deutliche Überlegenheit des CRAFT Ansatzes hinsichtlich der Rate an Behandlungsaufnahme (CRAFT: 58,6\% CRAFT + Nachsorge: 76,7\%) gegenüber der am 12-Schritte-Programm der Anonymen Alkoholiker ausgerichteten Al-Anon/Nar-Anon Behandlung [43].

\section{Ausblick}

Bislang existiert erst wenig Literatur zu der Nutzbarkeit familiärer Prozesse zur Initiierung und Aufrechterhaltung von Ausstiegsprozessen aus der Abhängigkeit, während verschiedene Studien eine klare Evidenz hinsichtlich des potenziell supportiven Einflusses familiärer Faktoren bereitstellen. Die Inanspruchnahme suchtbezogener Hilfeangebote durch Angehörige von Abhängigen ist demgegenüber sehr gering.

Mit dem CRAFT-Ansatz liegt ein neues, mit bewährten Interventionsverfahren kompatibles Modell vor, welches bislang in Deutschland keine Verbreitung gefunden hat. In kontrollierten Studien erwies sich dieser Ansatz als anderen Interventionsformen gegenüber deutlich überlegen. Die Replikation der Ergebnisse aus US-amerikanischen Studien und die Implementierung des Ansatzes in Deutschland erscheinen geboten. Es ist zu vermuten, dass eine zentrale Barriere hierfür in der Dominanz des empirisch nichtgestützten Co-Abhängigkeitskonzeptes liegen dürfte. Ein Paradigmenwechsel erscheint hier dringend geboten.

\section{Literatur}

\footnotetext{
${ }^{1}$ Copello AG, Velleman RDB, Templeton LJ. Family interventions in the treatment of alcohol and drug problems. Drug and Alcohol Review 2005; $24: 369-385$

2 Orford J, Guthrie S, Nicholls P et al. Self-reported coping behaviour of wives of alcoholics and its association with drinking outcome. Journal of Studies on Alcohol 1975; 36: 1254-1267

${ }^{3}$ Orford J, Templeton L, Velleman R et al. Family members of relatives with alcohol, drug and gambling problems: a set of standardized questionnaires for assessing stress, coping and strain. Addiction 2005; 100: $1611-1624$

${ }^{4}$ Velleman R, Bennett G, Miller T et al. The family of problem drug users: a study of 50 close relatives. Addiction 1993; 88: 1281 - 1289

${ }^{5}$ DHS (Hrsg.). Sucht und Familie. Freiburg: Lambertus, 1993

${ }^{6}$ Dittrich JE, Trappold MA. A treatment program for the wives of alcoholics: An evaluation. Bulletin of the Society of Psychologists in Addictive Behaviors 1984; 3: $91-102$
} 
7 Treating alcohol problems: Marital and family interventions. In: O'Farrell TJ (Hrsg). New York: Guilford, 1993

${ }^{8}$ Zweben A, Pearlman S. Evaluating the effectiveness of conjoint treatment of alcohol complicated marriages: Clinical and methodological issues. Journal of Marital and Family Therapy 1983; 9: 61 - 72

${ }^{9}$ Zweben A, Pearlman S, Li S. Reducing attrition from conjoint therapy with alcoholic couples. Drug and Alcohol Dependence 1983; 11: $321-331$

${ }^{10}$ Rumpf HJ, Bischof G, Hapke U et al. The role of family and partnership in recovery from alcohol dependence: comparison of individuals remitting without formal help and with and without formal help. European Addiction Research 2002; 8: 122 - 127

11 Blume A. Women and alcohol. JAMA 1986; 256: 1467-1470

${ }^{12}$ Bischof G, Rumpf HJ, Hapke U et al. Gender differences in natural recovery from alcohol dependence. Journal of Studies on Alcohol 2000; 61: $783-786$

${ }^{13}$ Reich G, Riehl-Emde A. Familientherapie: Aktuelle Trends und Diskussionen. Psychotherapeut 2001; 46: 355-367

14 O'Farrell TJ. Behavioral couples therapy for alcoholism and drug abuse. Psychiatric Times 1999; 16

15 Grant BF. Toward an alcohol treatment model: a comparison of treated and untreated respondents with DSM-IV alcohol use disorders in the general population. Alcoholism: Clinical and Experimental Research 1996; 20: $372-378$

${ }^{16}$ Rumpf HJ, Meyer C, Hapke U et al. Inanspruchnahme suchtspezifischer Hilfen von Alkoholabhängigen und -mißbrauchern: Ergebnisse der TACOS Bevölkerungsstudie. Sucht 2000; 46: 9-17

${ }_{17}$ Welsch K. Jahresstatistik der professionellen Suchtkrankenhilfe In: Deutsche Hauptstelle für Suchtfragen (Hrsg). Jahrbuch Sucht 2003. Geesthacht: Neuland, 2003

${ }^{18}$ Bischof G. Ausstiegsprozesse aus der Alkoholabhängigkeit mit und ohne formelle Hilfen. Ein Geschlechtervergleich. Freiburg: Lambertus, 2004

${ }^{19}$ Miller WR, Meyers RJ, Tonigan JS. Engaging the unmotivated in treatment for alcohol problems: a comparison of three strategies for intervention through family members. Journal of Consulting and Clinical Psychology 1999; 67: 688-697

${ }^{20}$ Holder H, Longabaugh R, Miller W et al. The cost effectiveness of treatment for alcoholism: a first approximation. Journal of Studies on Alcohol 1991; 52: $517-540$

${ }^{21}$ Miller WR, Wilbourne PL. Mesa Grande: a methodological analysis of clinical trials of treatments for alcohol use disorders. Addiction 2002; 97: $265-277$

22 Sisson RW, Azrin NH. Family-member involvement to initiate and promote treatment of problem drinkers. Behavior Therapy and Experimental Psychiatry 1986; 17: 15-21

${ }^{23}$ Cunningham JA, Sobell LC, Sobell MB et al. Resolution from alcohol problems with and without treatment: Reasons for change. Journal of Substance Abuse 1995; 7: 365-372

${ }^{24}$ Smith JE, Meyers RJ. Motivating substance abusers to enter treatment. Working with family members New York: Guilford, 2004

${ }^{25}$ Meyers RJ, Wolfe BL. Get your Loved One sober. Alternatives to nagging, pleading, and threatening. Minnesota: Hazelden, 2004

${ }^{26}$ Bischof G, Rumpf HJ, Meyer C et al. Änderungsmotivation In: Thomasius R and Küstner UJ (Hrsg). Familie und Sucht. Grundlagen, Therapiepraxis, Prävention. Stuttgart: Schattauer, 2005
${ }^{27}$ Miller WR, Rollnick S. Motivierende Gesprächsführung. 2. Auflage. Freiburg: Lambertus, 2004

${ }^{28}$ Tuschen B. Problemanalyse In: Margraf J (Hrsg). Lehrbuch der Verhaltenstherapie, Band 1, Grundlagen, Diagnostik, Verfahren, Rahmenbedingungen. 2., vollständig überarbeitete und erweiterte Auflage. Berlin: Springer, 2000: 291-299

${ }^{29}$ Lipsey MW, Wilson DB, Cohen MA et al. Is there a causal relationship between alcohol use and violence? A synthesis of the evidence. In: Galanter M (Hrsg). Recent developments in alcoholism. Alcohol and Violence: Epidemiology, neurobiology, psychology, and family issues. New York: Plenum Press, 1997: 245-282

${ }^{30}$ White HR, Chen P. Problem drinking and intimate partner Violence. Journal of Studies on Alcohol 2002; 63: 205-214

${ }^{31}$ Sullivan CM, Basta J, Tan C et al. After the crisis: A needs assessment of women leaving a domestic shelter. Violence and Victims 1992; 7: 267-275

32 Epstein EE, McCrady BS. Behavioral couples treatment of alcohol and drug use disorders: Current status and innovations. Clinical Psychology Review 1998; 18: 689-711

33 Pfingsten U. Training sozialer Kompetenz In: Margraf J (Hrsg). Lehrbuch der Verhaltenstherapie, Band 1, Grundlagen, Diagnostik, Verfahren, Rahmenbedingungen.2., vollständig überarbeitete und erweiterte Auflage. Berlin: Springer, 2000: 473-481

${ }^{34}$ D'Zurilla TJ, Goldfried MR. Problem solving and behavior modification. Journal of Abnormal Psychology 1971; 78: 107-126

35 Hautzinger M. Depression In: Margraf J (Hrsg). Lehrbuch der Verhaltenstherapie, 2. Auflage, Band 2. Berlin: Springer, 2000: 123-135

${ }^{36}$ Prochaska JO, DiClemente CC. Stages of change in the modification of addictive behaviors In: Hersen M, Eisler RM, and Miller PM (Hrsg). Progress in behavior modification. Pittsburgh: Sycamore Publishing Company, 1992: 3-67

37 Prochaska JO, DiClemente CC. Toward a comprehensive model of change In: Miller WR and Heather N (Hrsg). Treating addictive behaviors: Processes of change. New York: Plenum Press, 1986: 3-27

38 Diagnostic and statistical manual of mental disorders, fourth edition, international version. In: American Psychiatric Association (Hrsg). Washington D.C.: American Psychiatric Association, 1995

${ }^{39}$ Johnson VE. Intervention: How to help those who don't want help Minneapolis: Johnson Institute, 1986

${ }^{40}$ Liepman MR, Nirenberg TD, Begin AM. Evaluation of a program designed to help family and significant others to motivate resistant alcoholics into recovery. American Journal of Drug and Alcohol Abuse 1989; 15: $209-221$

${ }^{41}$ Hasin DS. Treatment/self-help for alcohol-related problems: Relationship to social pressure and alcohol dependence. Journal of Studies on Alcohol 1994; 55: 660-666

42 Bischof G, Rumpf HJ, Meyer C et al. Vergleich auslösender Faktoren für Remission ohne formelle Hilfen und Inanspruchnahme stationärer Behandlung. Sucht 2000; 46: 54-61

${ }^{43}$ Meyers RJ, Miller WR, Smith JE et al. A randomized trial of two methods for engaging treatment-refusing drug users through concerned significant others. Journal of Consulting and Clinical Psychology 2002; 70: $1182-1185$ 